\title{
CircMAPK9 promotes the progression of fibroblast-like synoviocytes in rheumatoid arthritis via the miR-140-3p/PPM1A axis
}

\author{
Zhihuan Luo ${ }^{\dagger}$, Shaojian Chen ${ }^{*}$ and Xiaguang Chen
}

\begin{abstract}
Background: Rheumatoid arthritis (RA) is a chronic inflammatory joint disease, and fibroblast-like synoviocytes (FLSs) are key effector cells in RA development. Mounting evidence indicates that circular RNAs (circRNAs) participate in the occurrence and development of RA. However, the precise mechanism of circRNA mitogenactivated protein kinase (circMAPK9) in the cell processes of FLSs has not been reported.
\end{abstract}

Methods: The expression levels of circMAPK9, microRNA-140-3p (miR-140-3p), and protein phosphatase magnesium-dependent 1A (PPM1A) were determined by quantitative real-time polymerase chain reaction (qRTPCR) or western blot assay. Cell proliferation was examined by 3-(4,5-dimethylthiazol-2-yl)-2,5-diphenyltetrazolium bromide (MTT) assay. Cell apoptosis and cycle distribution were assessed by flow cytometry. Cell migration and invasion were tested by transwell assay. All the proteins were inspected by western blot assay. Inflammatory response was evaluated by enzyme-linked immunosorbent assay (ELISA). The interaction between miR-140-3p and circMAPK9 or PPM1A was verified by dual-luciferase reporter assay.

Results: CircMAPK9 and PPM1A were upregulated and miR-140-3p was downregulated in RA patients and FLSs from RA patients (RA-FLSS). CircMAPK9 silence suppressed cell proliferation, migration, invasion, inflammatory response, and promoted apoptosis in RA-FLSs. MiR-140-3p was a target of circMAPK9, and miR-140-3p downregulation attenuated the effects of circMAPK9 knockdown on cell progression and inflammatory response in RA-FLSs. PPM1A was targeted by miR-140-3p, and circMAPK9 could regulate PPM1A expression by sponging miR140-3p. Furthermore, miR-140-3p could impede cell biological behaviors in RA-FLSs via targeting PPM1A.

Conclusion: CircMAPK9 knockdown might inhibit cell proliferation, migration, invasion, inflammatory response, and facilitate apoptosis in RA-FLSs via regulating miR-140-3p/PPM1A axis, offering a new mechanism for the comprehension of RA development and a new insight into the potential application of circMAPK9 in RA treatment.

Keywords: Rheumatoid arthritis, Fibroblast-like synoviocytes, circMAPK9, miR-140-3p, PPM1A

\section{Introduction}

Rheumatoid arthritis (RA) is a common chronic autoimmune disorder that mainly influences the synovial joints $[1,2]$. Fibroblast-like synoviocytes (FLSs), one

\footnotetext{
* Correspondence: mgk8oo@126.com

†Zhihuan Luo and Shaojian Chen contributed equally to this work. Department of Sports Medical, The Affiliated Ganzhou Hospital of Nanchang University, Ganzhou People's Hospital, No.17 Hongqi Avenue, Zhanggong District, Ganzhou City 341000, Jiangxi Province, China
}

class of dominating cells in synovial tissues, are reported to serve a vital role in the pathogenesis of RA [3, 4]. Although several treatment options are available for the management of RA patients, there is no cure for RA [5]. Thus, it is essential to explore the mechanism of FLSs progression in order to find new targets for RA treatment.

\section{$\triangle B M C$}

(c) The Author(s). 2021 Open Access This article is licensed under a Creative Commons Attribution 4.0 International License, which permits use, sharing, adaptation, distribution and reproduction in any medium or format, as long as you give appropriate credit to the original author(s) and the source, provide a link to the Creative Commons licence, and indicate if changes were made. The images or other third party material in this article are included in the article's Creative Commons licence, unless indicated otherwise in a credit line to the material. If material is not included in the article's Creative Commons licence and your intended use is not permitted by statutory regulation or exceeds the permitted use, you will need to obtain permission directly from the copyright holder. To view a copy of this licence, visit http://creativecommons.org/licenses/by/4.0/ The Creative Commons Public Domain Dedication waiver (http://creativecommons.org/publicdomain/zero/1.0/) applies to the data made available in this article, unless otherwise stated in a credit line to the data. 
Circular RNAs (circRNAs) are a special class of noncoding RNAs possessing continuous covalently closed loops that are produced via back-splicing of precursor mRNAs [6, 7]. Increasing evidence supported that circRNAs are concerned with the onset and development of multiple human diseases [8]. Meanwhile, numerous circRNAs played vital part in the progression of autoimmune diseases, including RA [9]. CircRNA mitogenactivated protein kinase (circMAPK9), also known as hsa_circ_0001566, is derived from back-splicing of MAPK9 transcript and has been reported to be highly expressed in peripheral blood mononuclear cells (PBMCs) from RA patients [10], whereas the exact role and regulatory mechanism of circMAPK9 in FLSs progression is indistinct.

MicroRNAs (miRNAs) are defined as small noncoding molecules that can regulate gene expression through combining with the 3'untranslated regions (3'UTRs) of target mRNAs [11]. Generally, circRNAs are known to work as miRNA molecular sponges to inhibit miRNA activity by competitively binding to miRNAs [12]. Plentiful miRNAs have been found to be dysregulated and may serve as biomarkers or therapeutic targets in RA [13-15]. Furthermore, miR-140-3p abundance was declined in synovial tissue and FLSs from RA patients (RAFLSs) and from mice in arthritis models, and miR-140$3 p$ overexpression in FLSs inhibited cell proliferation and migration [16]. Moreover, Lee et al. reported that protein phosphatase magnesium-dependent 1A (PPM1A) was involved in the development of RA [17]. Nevertheless, the relationships among circMAPK9, miR140-3p, and PPM1A in the pathogenesis of RA are undiscovered.

In this research, circMAPK9 abundance was measured in RA patients and RA-FLSs. Then, we explored the effects of circMAPK9 on cell growth, transferability, and inflammation in RA-FLSs. Besides, we uncovered the regulatory network of circMAPK9/miR-140-3p/PPM1A in RA-FLSs.

\section{Materials and methods \\ Patient tissue collection}

RA synovial tissues were collected from RA patients $(n=$ 22) who underwent knee replacement surgery. Normal synovial tissues were obtained from patients with traumatic knee and no history of autoimmune diseases $(n=$ 22). All subjects were recruited from Ganzhou People's Hospital, The Affiliated Ganzhou Hospital of Nanchang University, and they all signed the written informed consent. After surgical resection, these tissues were preserved at $-80{ }^{\circ} \mathrm{C}$ until usage. This research was permitted by the Ethical Committee of Ganzhou People's Hospital, The Affiliated Ganzhou Hospital of
Nanchang University. The clinical characteristics of RA and trauma patients are listed in Table 1.

\section{Cell culture}

Fibroblast-like synoviocytes from RA patients (RA-FLSs) or healthy subjects (H-FLSs) were separated as formerly mentioned [18]. Briefly, synovial tissue samples were cut into small debris and digested using $2 \mathrm{mg} / \mathrm{mL}$ of collagenase (type II, Thermo Fisher Scientific, Waltham, MA, USA) at $37{ }^{\circ} \mathrm{C}$ for $2 \mathrm{~h}$ to isolate synoviocytes. FLSs were cultured in HFLS growth medium (Cell Applications, San Diego, CA, USA) plus 10\% fetal bovine serum (FBS, HyClone, Logan, UT, USA) in a $37{ }^{\circ} \mathrm{C}$ incubator containing $5 \% \mathrm{CO}_{2}$. FLSs were separated from all healthy donors and RA patients for detecting the circMAPK9 expression. Two sets of RA-FLSs were selected for functional assays. Set 1 of RA-FLSs was acquired from three random-selected RA patients and set 2 of RA-FLS was acquired from another three random-selected RA patients. Then, the same number of RA-FLSs was mixed from these three RA patients. The RA-FLSs were cultured, and set 1 of RA-FLSs was utilized for mechanistic investigation. In these experiments, cells at passage 3 were used.

\section{Quantitative real-time polymerase chain reaction (qRT- PCR)}

Total RNA was extracted with TRIzol (Invitrogen, Carlsbad, CA, USA). Next, cDNA was synthesized from RNA reverse transcription using M-MLV Reverse

Table 1 Clinical characteristics of rheumatoid arthritis (RA) and trauma patients

\begin{tabular}{|c|c|c|}
\hline Clinical data & RA patients & Trauma patients \\
\hline Number of subjects & 22 & 22 \\
\hline Sex (male/female) & $10 / 12$ & $8 / 14$ \\
\hline Age (years) & $51.35 \pm 15.25$ & $46.54 \pm 11.32$ \\
\hline Disease duration (years) & $8.65 \pm 5.68$ & / \\
\hline Tender joints ${ }^{a}$ & $10.35 \pm 9.56$ & / \\
\hline Swollen joints ${ }^{a}$ & $11.68 \pm 9.86$ & / \\
\hline $\operatorname{CRP}(\mathrm{mg} / \mathrm{dl})$ & $34.25 \pm 23.65$ & / \\
\hline $\mathrm{ESR}(\mathrm{mm} / \mathrm{h})$ & $42.35 \pm 25.68$ & / \\
\hline ACPA (RU/ml) & $712.65 \pm 512.34$ & / \\
\hline DAS28 & $5.48 \pm 1.95$ & / \\
\hline DMARD (n) & 14 & / \\
\hline NSAID (n) & 9 & / \\
\hline Corticosteroid (n) & 12 & \\
\hline
\end{tabular}

$C R P$ C-reactive protein, ESR erythrocyte sedimentation rate, $A C P A$ anticitrullinated protein antibodies, DAS28 disease activity score, DMARD diseasemodifying anti-rheumatic drug, NSAID non-steroidal anti-inflammatory drug, $n$ number of patients

${ }^{a}$ Twenty-eight joints were assessed for tenderness, and twenty-eight were assessed for swelling 
Transcriptase kit (Thermo Fisher Scientific) or TaqMan microRNA Reverse Transcription Kit (Applied Biosystems, Foster City, CA, USA). Then, qRT-PCR were implemented on ABI Prism 7900HT Detection System (Applied Biosystems) employing SYBR Master Mix (Takara, Tokyo, Japan) and specific primers (Sangon, Shanghai, China) with the amplification protocol: $95{ }^{\circ} \mathrm{C}$ for $5 \mathrm{~min}, 40$ cycles of $95^{\circ} \mathrm{C}$ for $30 \mathrm{~s}, 55^{\circ} \mathrm{C}$ for $45 \mathrm{~s}$, and $72{ }^{\circ} \mathrm{C}$ for $30 \mathrm{~s}$. The relative RNA expression was calculated with $2^{-\Delta \Delta \mathrm{Ct}}$ method. The primers were exhibited as follows: circMAPK9 (F, 5'-CATGGAGCTGGATC ATGAAA-3'; R, 5'-AGGTTGAGTCTGCCACTTGC3'), MAPK9 (F, 5' -TACGTGGTGACACGGTACTACC3'; R, 5'-CACAACCTTTCACCAGCTCTCC-3'), miR140-3p (F, 5'-CAGTGCTGTACCACAGGGTAGA-3'; R, 5'-TATCCTTGTTCACGACTCCTTCAC-3'), PPM1A (F, 5'-GAAGAAGGAGGCAGAGTTGGAC-3'; R, 5'-GGATGTTCTCACTCGCTAATGTG-3'), U6 (F, 5' -CTCGCTTCGGCAGCACA-3'; R, 5'-AACGCTTC ACGAATTTGCGT-3'), and $\beta$-actin (F, $5^{\prime}$-GTCACCGG AGTCCATCACGAT-3'; R, 5'-TCACCAACTG GGACGACATG-3'). $\beta$-actin and U6 were served as the inner references.

\section{RNase R treatment}

For detecting the stability of circMAPK9, total RNA (2 $\mu \mathrm{g})$ was reacted with RNase $\mathrm{R}(3 \mathrm{U} / \mu \mathrm{g}$, Geneseed, Guangzhou, China) at $37{ }^{\circ} \mathrm{C}$ for $0.5 \mathrm{~h}$ to digest linear RNA. After that, the treated RNA was used for qRTPCR to survey the RNA abundance of circMAPK9 and linear MAPK9.

\section{Cell transfection}

The small interfering RNA (siRNA) against circMAPK9 (si-circMAPK9\#1, si-circMAPK9\#2 or si-circMAPK9\#3), miR-140-3p mimic (miR-140-3p) or miR-140-3p inhibitor (anti-miR-140-3p), and corresponding controls (si$\mathrm{NC}$, miR-NC or anti-miR-NC) were acquired from Ribobio (Guangzhou, China). PPM1A overexpression vector (PPM1A) based on the pcDNA3.1 vector and the empty pcDNA3.1 vector (vector) were also acquired from Ribobio. Cell transfection was performed through introducing the above oligonucleotides $(50 \mathrm{nM})$ or vectors $(40$ ng) into RA-FLSs $\left(1 \times 10^{5}\right)$ at $70-80 \%$ confluence via the Lipofectamine 3000 reagent (Invitrogen; $1 \mu \mathrm{L} /$ each well).

\section{3-(4,5-Dimethylthiazol-2-yl)-2,5-diphenyltetrazolium bromide (MTT) assay}

For proliferation detection, $1 \times 10^{4}$ RA-FLSs were placed into 96-well plates and incubated for $72 \mathrm{~h}$. Then, the cells were shifted into fresh medium plus MTT $(0.5 \mathrm{mg} /$ $\mathrm{mL}$, Beyotime, Shanghai, China). After incubation for another $4 \mathrm{~h}$, the medium was changed to $100 \mu \mathrm{L}$ dimethyl sulfoxide (DMSO; Solarbio, Beijing, China). The absorbance at $570-\mathrm{nm}$ wavelength was examined via a microplate reader (Molecular Devices, Sunnyvale, CA, USA).

\section{Flow cytometry}

Flow cytometry analysis was executed for the detection of cell apoptosis and cycle distribution. For cell apoptosis detection, Annexin V-fluorescein isothiocyanate (FITC) Apoptosis Detection Kit (BD Biosciences, San Jose, CA, USA) was utilized. Briefly, following transfection for $48 \mathrm{~h}, 1 \times 10^{5}$ RA-FLSs were collected and resuspended in Annexin binding buffer. Then, cells were stained with $5 \mu \mathrm{L}$ Annexin V-FITC and $5 \mu \mathrm{L}$ propidium iodide (PI) in a dark place at $4{ }^{\circ} \mathrm{C}$ for $15 \mathrm{~min}$. Subsequently, the apoptotic cells were analyzed by a flow cytometer (BD Biosciences). For cell cycle distribution detection, RA-FLSs $\left(1 \times 10^{5}\right)$ after 48 -h transfection were collected and re-suspended in PBS, then added with PI (BD Biosciences) staining solution in dark place at $37{ }^{\circ} \mathrm{C}$ for $10 \mathrm{~min}$ after $70 \%$ ethanol fixation. The distribution of different cell cycle phases (G0/G1, S and $\mathrm{G} 2 / \mathrm{M}$ ) was assessed utilizing a flow cytometer (BD Biosciences).

\section{Transwell assay}

Transwell chambers (Costar, Corning, NY, USA) were used to detect cell migratory and invasive abilities. $5 \times$ $10^{4}$ RA-FLSs or $1 \times 10^{4}$ RA-FLSs after 48 -h transfection was re-suspend in $200 \mu \mathrm{L}$ of serum free HFLS medium and then added into the upper chamber coated with or without Matrigel (BD Biosciences) to identify cell invasion and migration, respectively. The lower chamber was added with $0.6 \mathrm{~mL}$ complete HFLS medium with $10 \%$ FBS. The non-migrating or non-invading cells were removed after incubation for $24 \mathrm{~h}$, and migratory and invasive cells on the bottom membrane were fastened by paraformaldehyde (4\%, Beyotime) and dyed by crystal violet solution ( $0.1 \%$, Beyotime) for $30 \mathrm{~min}$. The migratory or invasive cells were observed and counted using a microscope (Olympus, Tokyo, Japan) at $\times 100$ magnification.

\section{Western blot}

RIPA lysis buffer (Beyotime) and BCA protein assay kit (Beyotime) were utilized for total protein extraction and quantification, respectively. Then, equal amount of protein $(20 \mu \mathrm{g} / \mathrm{lane})$ was segregated by SDS-PAGE gel and then shifted into PVDF membrane (Solarbio). After blocked in $5 \%$ non-fat milk for $1 \mathrm{~h}$, the membrane was probed with specific primary antibodies at $4{ }^{\circ} \mathrm{C}$ overnight, and subsequently probed with HRP-conjugated secondary antibody (1:20,000, ab205718 or ab205719, Abcam, Cambridge, MA, USA) for $2 \mathrm{~h}$. The blots were 
exposed to enhanced BeyoECL Moon (Beyotime), and the bands density was assessed via Image I software (NIH, Bethesda, MD, USA). The primary antibodies obtained from Abcam included anti-B-cell lymphoma-2 antibody (Bcl-2; 1:1000, ab32124), anti-BCL2-associated $\mathrm{X}$ protein antibody (anti-Bax; 1:1000, ab32503), antimatrix metalloproteinase 2 (MMP2) (1:1000, ab86607) antibody, anti-MMP9 (1:1000, ab137867) antibody, antiPPM1A (1:500, ab14824) antibody, and $\beta$-actin antibody (1:5000; ab6276). Relative protein expression was normalized by internal reference $\beta$-actin.

\section{Enzyme-linked immunosorbent assay (ELISA)}

ELISA was performed to inspect the secretion of tumor necrosis factor alpha (TNF- $\alpha$ ), interleukin (IL)-1 $\beta$ and IL-6 in culture supernatant of RA-FLSs. Briefly, $1 \times 10^{5}$ RA-FLSs were plated into 12-well plates. Seventy-two hours post-transfection, culture supernatant of each group was collected. The concentrations of TNF- $\alpha$, IL$1 \beta$, and IL- 6 in culture supernatant were examined using corresponding Human TNF- $\alpha$, IL-1 $\beta$, or IL-6 ELISA Kit (Abcam).

\section{Dual-luciferase reporter analysis}

The sequence of circMAPK9 or PPM1A 3'UTR carrying wild-type (WT) or mutant (MUT) complementary sites of miR-140-3p was cloned into pmirGLO vector (Promega, Madison, WI, USA) to generate circMAPK9 WT and circMAPK9 MUT, PPM1A 3'UTR WT, and PPM1A 3'UTR MUT. The sites of circMAPK9 and PPM1A were mutated using the QuickChange XL site-directed mutagenesis kit (Stratagene, La Jolla, CA, USA). RA-FLSs $\left(1 \times 10^{5}\right.$ cells $)$ were transfected with the constructed luciferase vector $(20$ $\mathrm{ng})$ and miR-NC or miR-127-5p (20 nM) for $48 \mathrm{~h}$. The luciferase activity was examined by Dual-Lumi ${ }^{\text {mo }}$ Luciferase Assay Kit (Beyotime), followed by normalization to the Renilla luciferase.

\section{Statistical analysis}

Statistical analysis was executed using GraphPad Prism 6 (GraphPad Inc., La Jolla, CA, USA). All data from at least 3 independent biological replications were displayed as mean \pm standard deviation (SD). Difference was analyzed using Student's $t$-test (between 2 groups) or one-way analysis of variance followed by Tukey test (among multiple groups) in specific circumstances. Statistical significance was considered when $P$-value $<0.05$.

\section{Results}

CircMAPK9 was upregulated in RA patients and RA-FLSs CircMAPK9 (hsa_circ_0001566) was located on chr5: 179688683-179707608 of chromosome and derived from exon 16-21 of MAPK9 genome (Fig. 1A). To explore the potential roles of circMAPK9 in RA, its expression pattern was detected by qRT-PCR in synovial tissues from RA patients $(n=22)$ and normal patients $(n=22)$. The results showed that circMAPK9 abundance was greatly increased in RA patients compared to normal patients (Fig. 1B). Then, the expression of circMAPK9 in RAFLSs or normal subjects (H-FLSs) was measured. The results demonstrated that circMAPK9 level was higher in RA-FLSs more than triple than control group (Fig. $1 C)$. Furthermore, the stability of circRNA was evaluated by RNase $\mathrm{R}$ digestion assay. As displayed in Fig. 1D, linear mRNA (MAPK9) was obviously decreased after digestion by RNase $\mathrm{R}$ while circMAPK9 expression was not affected, indicating the cyclic structure of circMAPK9. These data indicated that increased expression of circMAPK9 might be associated with RA progression.

\section{Knockdown of circMAPK9 inhibited cell proliferation, migration, invasion, inflammation, and promoted apoptosis in RA-FLSs}

To study the effect of circMAPK9 on RA progression, loss-of-function experiments were performed in RAFLSs transfected with siRNAs to knock down circMAPK9. As displayed in Fig. 2A, relative to si-NC group, the expression of circMAPK9 was signally

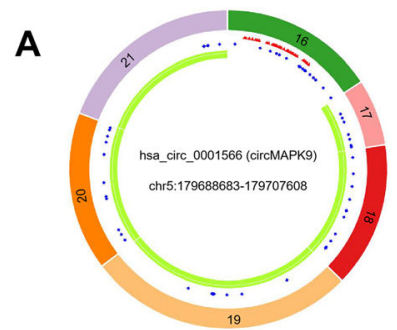

B

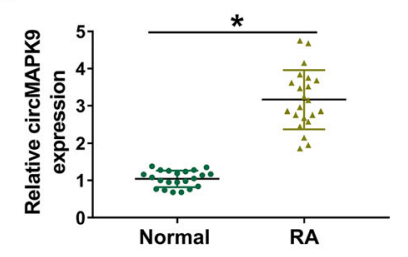

C

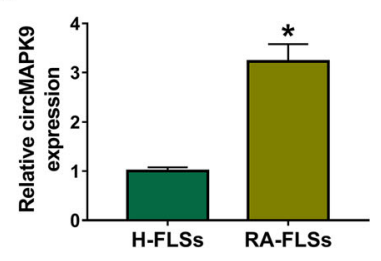

D

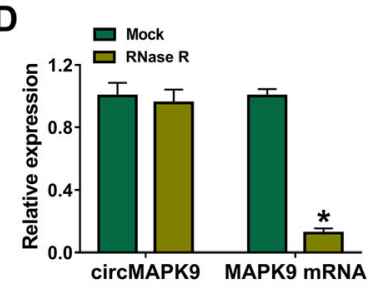

Fig. 1 CircMAPK9 expression was increased in RA patients and RA-FLSs. A Schematic illustration exhibiting the circularization of MAPK9 exons 16 to 21 forming circMAPK9. B The expression of circMAPK9 was determined by qRT-PCR analysis in synovial tissues from RA patients ( $n=22$ ) and normal patients $(n=22)$. C The abundance of circMAPK9 in RA-FLSs and control H-FLSs was measured via qRT-PCR. D The expression of circMAPK9 and linear mRNA (MAPK9) was examined via qRT-PCR after RNase $R$ treatment. * $P<0.05$ 


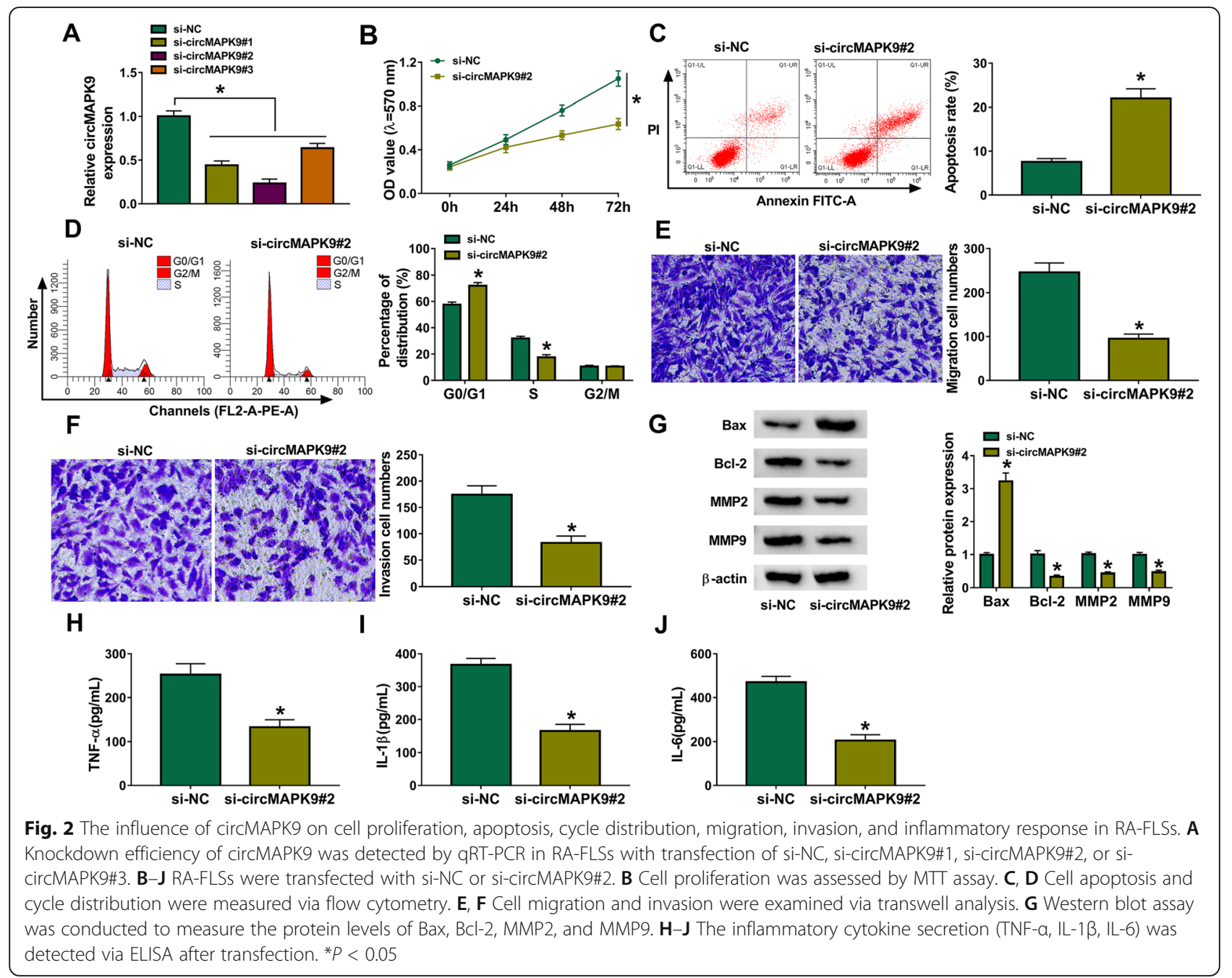

declined in RA-FLSs transfected with si-circMAPK9\#1, si-circMAPK9\#2, or si-circMAPK9\#3, especially in sicircMAPK9\#2 group. Therefore, si-circMAPK9\#2 was chosen for further study. Next, the impacts of circMAPK9 on cell proliferation, apoptosis, cycle distribution, and invasiveness were investigated. MTT assay indicated that knockdown of circMAPK9 restrained cell proliferation in RA-FLSs (Fig. 2B). Flow cytometry assay showed that circMAPK9 silence evidently promoted cell apoptosis and induced cell cycle arrest at G0/G1 phase in RA-FLSs (Fig. 2C, D). Moreover, circMAPK9 deficiency markedly restrained the migratory and invasive abilities of RA-FLSs using transwell analysis (Fig. 2E, F). Besides, western blot assay exhibited that circMAPK9 interference significantly increased the level of proapoptotic protein Bax, and decreased the expression of anti-apoptotic protein Bcl-2, migration and invasionrelated proteins (MMP2 and MMP9), further supporting the effects of circMAPK9 silence on cell apoptosis and invasiveness (Fig. 2G). Additionally, the inflammatory response was analyzed in RA-FLSs via ELISA, which presented that circMAPK9 knockdown visibly reduced the secretion of pro-inflammatory cytokines (TNF- $\alpha$, IL-1 $\beta$, and IL-6) in RA-FLSs (Fig. 2H-J). These results indicated that circMAPK9 downregulation could suppress cell proliferation, migration, invasion, inflammatory response, and accelerate apoptosis of RA-FLSs.

\section{CircMAPK9 acted as a sponge of miR-140-3p}

To analyze the potential mechanism of circMAPK9, the potential target miRNAs of circMAPK9 were predicted using CircInteractome (https://circinteractome.nia.nih. gov/mirna_target_sites.html). The results showed that miR-140-3p had putative binding sequence for circMAPK9 (Fig. 3A). To validate the relationship between circMAPK9 and miR-140-3p, dual-luciferase reporter assay was performed through constructing circMAPK9 WT and circMAPK9 MUT. The results displayed that miR-140-3p overexpression remarkably decreased the luciferase activity of circMAPK9 WT but not that of 


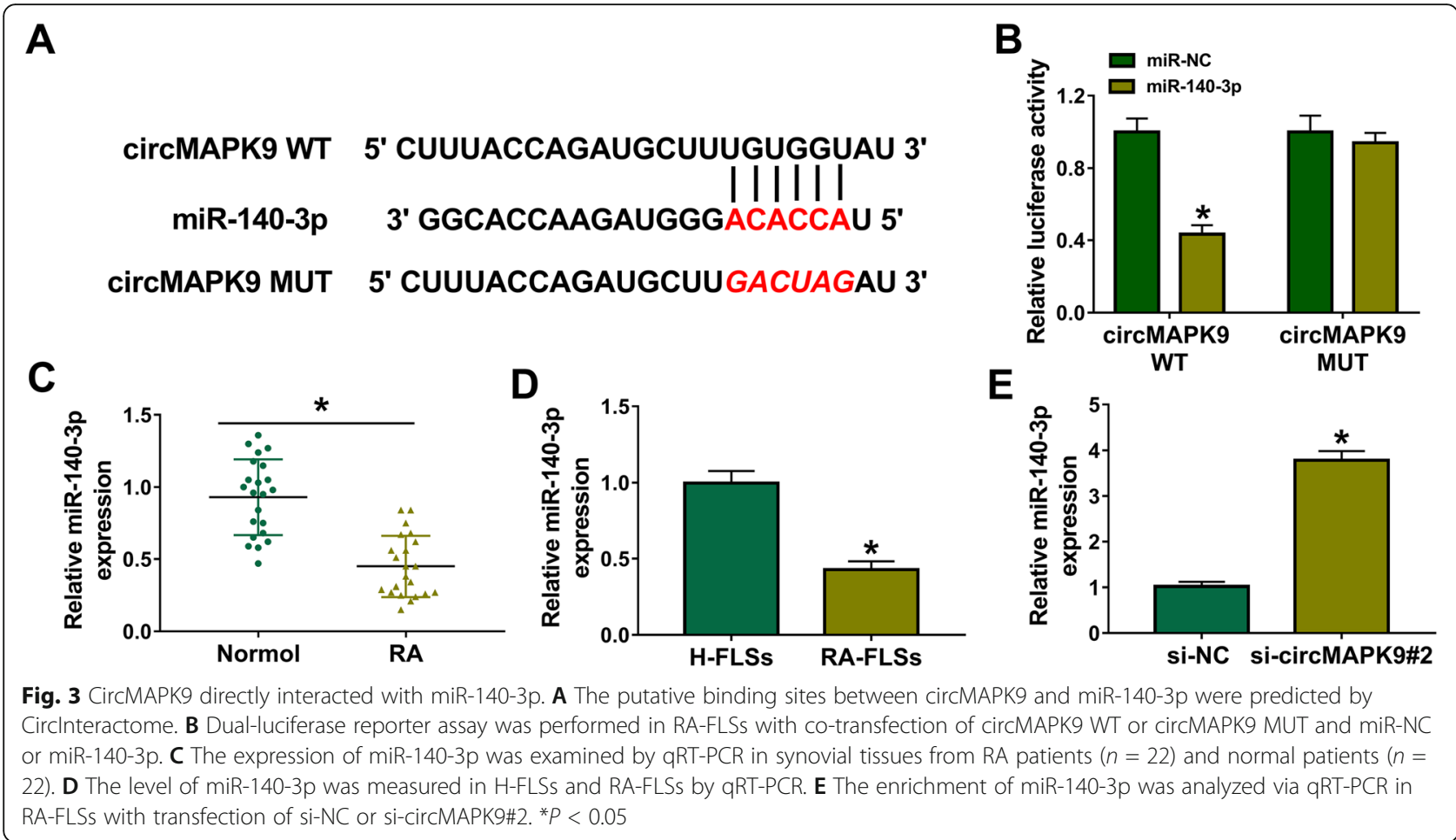

circMAPK9 MUT when the binding sites were mutated (Fig. 3B). Furthermore, the level of miR-140-3p in RA patients and RA-FLSs was observed. Results showed that the abundance of miR-140-3p was evidently decreased in synovial tissues from RA patients and RA-FLSs (Fig. 3C, D). In addition, the expression of miR-140-3p was promoted after knockdown of circMAPK9 (Fig. 3E). The above evidence verified that miR-140-3p was a direct target of circMAPK9.

MiR-140-3p knockdown reversed the effects of sicircMAPK9\#2 on cell proliferation, migration, invasion, and inflammatory response in RA-FLSs

To explore whether the biological function of circMAPK9 in RA was mediated by miR-140-3p, rescue experiments were performed. RA-FLSs were transfected with si-NC, sicircMAPK9\#2, si-circMAPK9\#2 + anti-miR-NC, or sicircMAPK9\#2 + anti-miR-140-3p. The abundance of miR-140-3p was increased via circMAPK9 knockdown, which was reversed by downregulating miR-140-3p (Fig. 4A). Furthermore, the inhibitory effect of circMAPK9 downregulation on cell proliferation and the promoting effects of circMAPK9 silence on apoptosis and cell cycle arrest at G0/G1 phase were all mitigated by downregulation of miR-140-3p (Fig. 4B-D). Moreover, miR-140-3p inhibition abated the effect of circMAPK9 silencemediated suppression on cell migration and invasion (Fig. $4 \mathrm{E}, \mathrm{F})$. Correspondingly, the enhancement of Bax expression and decrease of Bcl-2, MMP2, and MMP9 were all relieved by miR-140-3p deficiency in circMAPK9-silenced RA-FLSs (Fig. 4G). Additionally, the reduction of TNF- $\alpha$, IL-1 $\beta$, and IL-6 levels in RA-FLSs caused by sicircMAPK9\#2 transfection was restored by cotransfection with anti-miR-140-3p (Fig. 4H-J). Taken together, these data illustrated that circMAPK9 exerted its biological function in RA-FLSs by sponging miR-140-3p.

PPM1A was identified to be a target of miR-140-3p

To further analyze the regulatory network, starBase v2.0 online website (http://starbase.sysu.edu.cn/agoClipRNA. php? source $=$ mRNA) was utilized to search for the potential target mRNAs of miR-140-3p. The prediction result suggested that PPM1A 3'UTR shared binding sites for miR140-3p (Fig. 5A), suggesting that PPM1A could possibly interact with miR-140-3p. To confirm this assumption, PPM1A 3'UTR WT and PPM1A 3'UTR MUT were constructed, and then dual-luciferase reporter assay was implemented. The results indicated that miR-140-3p introduction significantly decreased the luciferase activity of PPM1A 3'UTR WT, whereas little change was observed in the luciferase activity of PPM1A 3'UTR MUT (Fig. 5B). The qRT-PCR and western blot assays results displayed that PPM1A mRNA and protein abundance was markedly upregulated in synovial tissues from RA patients and RAFLSs (Fig. 5C-F). Next, the relationships among circMAPK9, miR-140-3p, and PPM1A were explored. The result of qRT-PCR showed that the abundance of miR-140$3 p$ was strikingly increased in RA-FLSs transfected with 


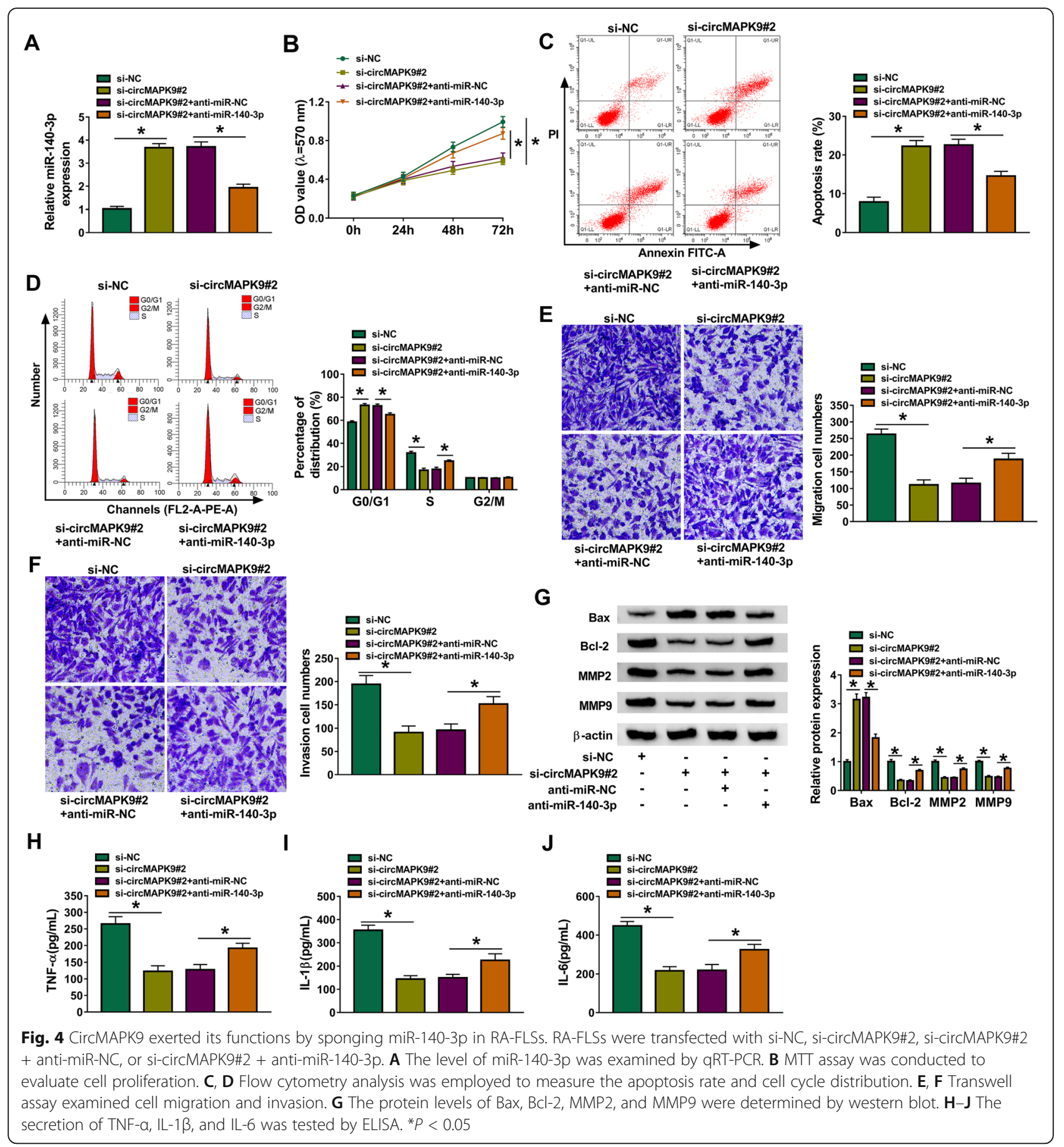

miR-140-3p (Fig. 5G), indicating the high transfection efficacy of miR-140-3p. Meanwhile, overexpression of miR$140-3 p$ visibly inhibited the mRNA and protein expression of PPM1A (Fig. 5H, I). Moreover, circMAPK9 silence markedly reduced the mRNA and protein levels of PPM1A, which could be reversed by downregulating miR-140-3p (Fig. 5J, K), indicating that circMAPK9 upregulated PPM1A expression by downregulating miR-140-3p. These data collectively demonstrated that PPM1A was a downstream target of miR-140-3p, and circMAPK9 could positively regulate PPM1A expression by sponging miR-140-3p.

\section{MiR-140-3p overexpression could suppress cell progression and inflammatory response via downregulating PPM1A in RA-FLSs}

To explore whether miR-140-3p exerted its biological functions in RA-FLSs by targeting PPM1A, RA-FLSs were transfected with miR-NC, miR-140-3p, miR-140-3p 


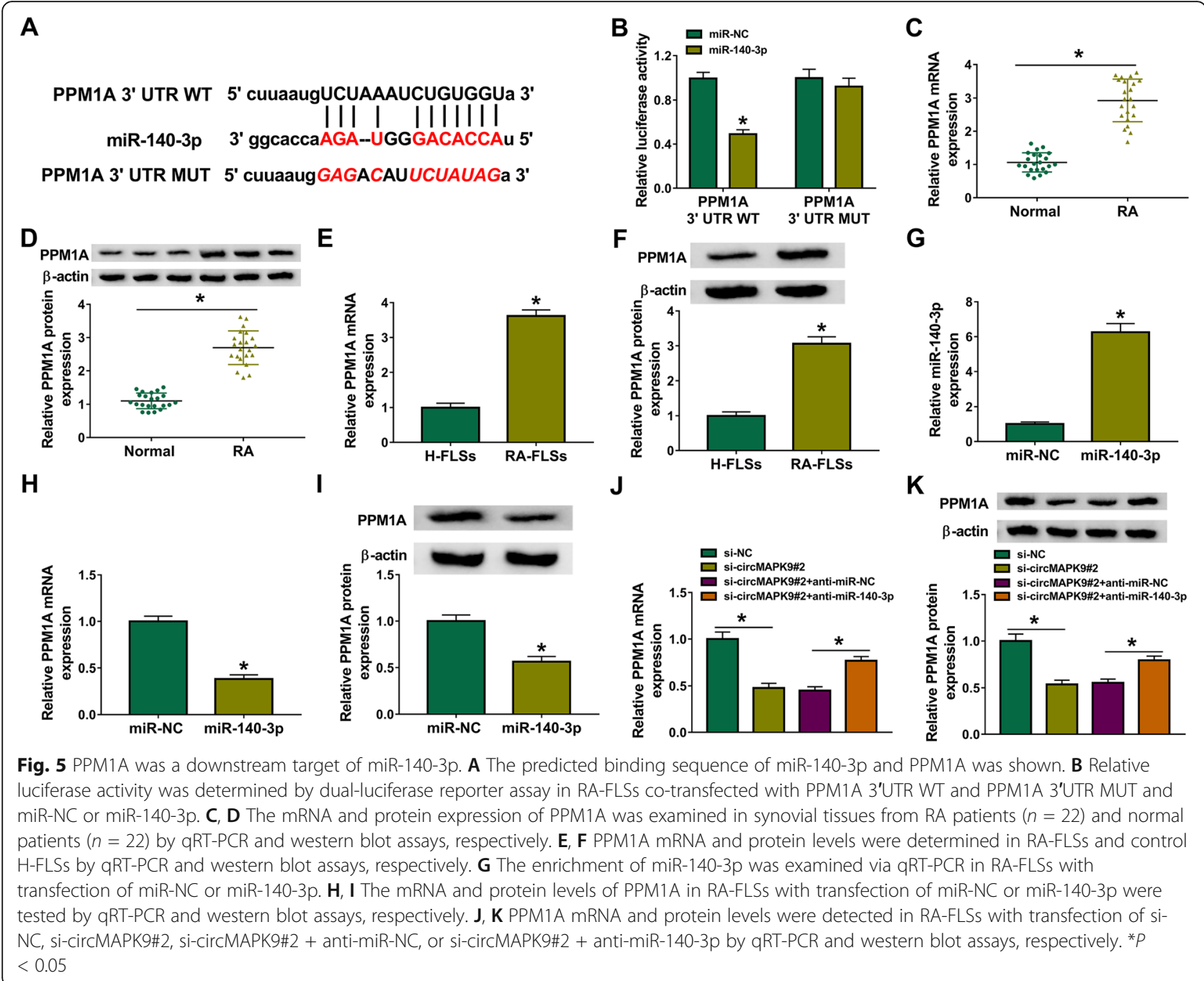

+ vector, or miR-140-3p + PPM1A. Western blot assay showed that miR-140-3p overexpression overtly inhibited the protein expression of PPM1A, which was restored via addition of PPM1A overexpression vector (Fig. 6A). Moreover, overexpression of miR-140-3p repressed cell proliferation and inducing cycle arrest at G0/G1 phase and apoptosis, which could be reversed by introduction of PPM1A (Fig. 6B-D). Also, PPM1A upregulation evidently overturned the repressive effect of miR-140-3p overexpression on cell migration and invasion (Fig. 6E, F). Furthermore, miR-140-3p addition increased the protein level of $\mathrm{Bax}$ and decreased the protein expression of $\mathrm{Bcl}-2$ and $\mathrm{MMP} 2$ as well as MMP9, whereas these effects were abated by upregulating PPM1A (Fig. 6G). Additionally, the protein levels of TNF- $\alpha$, IL-1 $\beta$, and IL- 6 were significantly reduced in RA-FLSs after transfection with miR-140-3p, while cotransfection with PPM1A overexpression vector mitigated these effects (Fig. 6H-J). Altogether, these data proved that miR-140-3p could inhibit cell proliferation, migration, invasion, inflammatory response, and facilitate apoptosis of RA-FLSs by targeting PPM1A.

\section{Discussion}

RA is a common form of inflammatory multisystem disease with undiscovered etiology [2]. CircMAPK9 was highly expressed in PBMCs from RA patients and might act as a possible diagnostic biomarker for RA [10]. FLSs play a pivotal role in RA etiology by regulating inflammatory response and cartilage destruction $[19,20]$. Thereby, investigating the tumor-like biologic behaviors of RA-FLSs is indispensable to develop novel therapies for RA patients. Meanwhile, noncoding RNAs (ncRNAs) including circRNAs might serve as promising biomarkers for RA [21]. In this report, we aimed to study the biological role of circMAPK9 and explore the underlying mechanism in the advancement of RA-FLSs. Through the verification of functional experiment, we first clarified that circMAPK9 knockdown repressed 


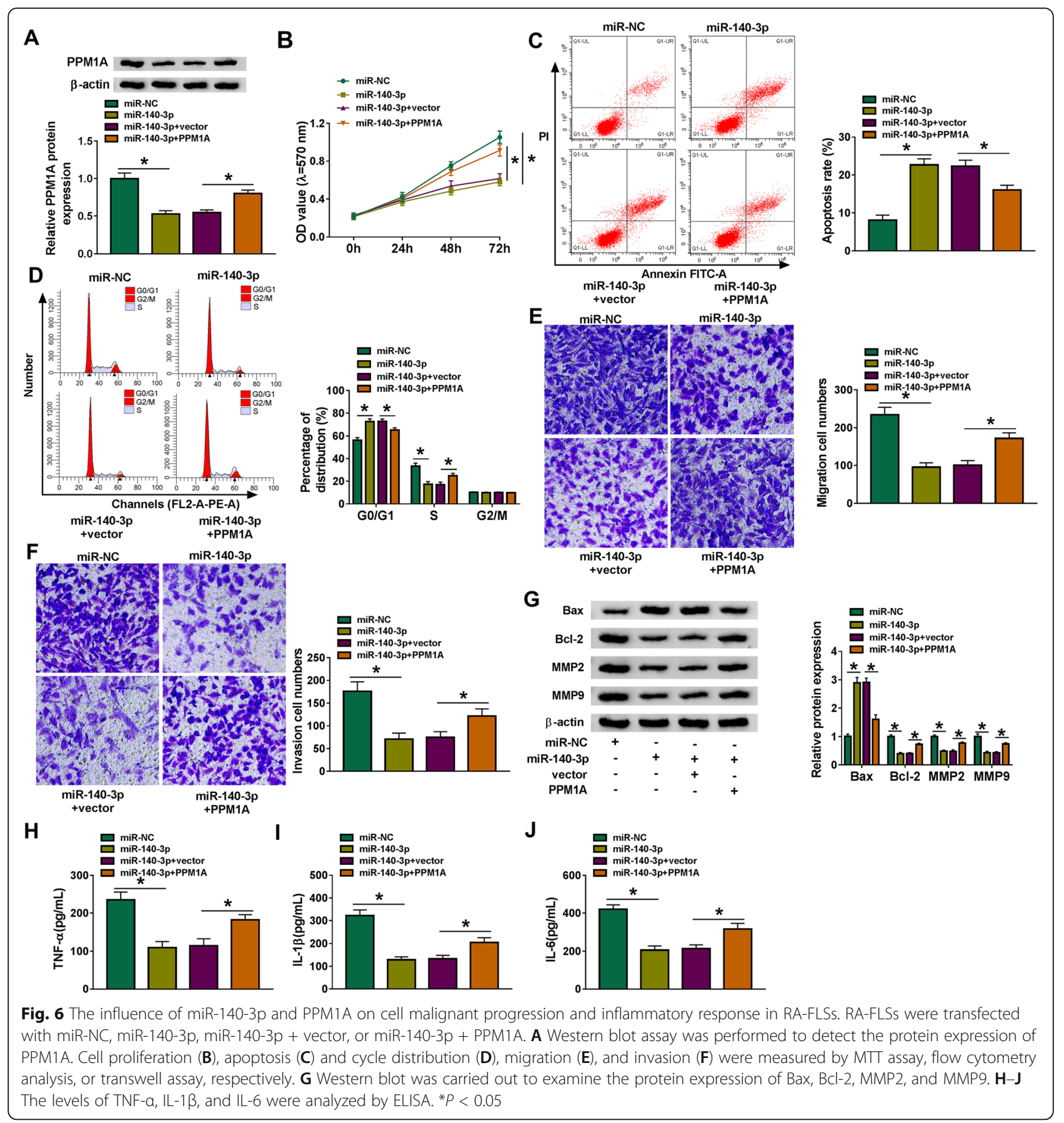

proliferation, invasiveness, and inflammation of RA-FLSs via circMAPK9/miR-140-3p/PPM1A regulatory network.

The deregulation of circRNAs is identified to be closely related to the occurrence and development of autoimmune diseases including RA [22]. For instance, circ_0088036 promoted the proliferative and migratory capacities of FLSs via the circ0088036/miR-140-3p/ SIRT1 axis in RA [23]. In keeping with previously report [10], we also verified that circMAPK9 level was enhanced in synovial tissues from RA patients and RA-
FLSs. Thus, we speculated that the disordered level of circMAPK9 might be connected with RA evolution. Through implementing loss-of-function experiment in RA-FLSs, it was evidenced that circMAPK9 silence could repress cell proliferation, migration, invasion, and accelerated apoptosis of RA-FLSs. Many cytokines are associated with RA progression, including TNF- $\alpha$, IL-1, IL-6, and IL-17 [24, 25]. Furthermore, this report also attested that circMAPK9 knockdown lessened the inflammatory response of RA-FLSs by decreasing the release of TNF- 
$\alpha$, IL-1 $\beta$, and IL- 6 . Hence, we deemed that circMAPK9 might contribute to RA malignant development by facilitating cell proliferation, migration, invasion, inflammatory response, and hindering cell apoptosis of RA-FLSs.

Accumulating reports have revealed that circRNAs could modulate the progression of multifarious diseases via acting as miRNA sponges [26]. As well, miRNAs have been certified to serve pivotal part in FLSs of RA [27]. For instance, miR-20a was involved in the modulation of pro-inflammatory cytokines release by controlling ASK1 expression in RA-FLSs [28]. To validate whether miRNAs were implicated in circMAPK9-mediated RA evolution, CircInteractome database was applied to forecast the possible miRNAs of circMAPK9. The prediction result indicated that miR-140-3p was targeted by circMAPK9 in RA-FLSs, and the dual-luciferase reporter assay verified the interacting effect between them furtherly. Previous studies have demonstrated the suppressive role of miR-140-3p in the progress of bladder cancer [29], colorectal cancer [30], and so on. Yin et al. pointed out that the decline of miR-140-3p was correlated with increased osteoarthritis severity [31]. Moreover, Zhong et al. illuminated the participation of miR$140-3 p$ in the proliferative and migratory processes of RA-FLSs via SIRT1 in RA [23]. In this research, low expression of miR-140-3p was observed in synovial tissues from RA patients and RA-FLSs, which was in agreement with previous work [16]. Simultaneously, miR-140-3p could restrain cell propagation, transferability, and inflammatory response of RA-FLSs. Besides, miR-140-3p silence restored the influence of circMAPK 9 deficiency on cell progression and inflammation in RA-FLSs. Therefore, these findings confirmed that circMAPK9 could modulate the aggressive phenotype of RA-FLSs by sponging miR-140-3p.

The circRNA/miRNA/mRNA network has been identified in diversiform diseases, such as hepatocellular carcinoma [32], gastric cancer [33], and systemic lupus erythematosus [34]. To explore the downstream mRNAs of circMAPK9/miR-140-3p network in RA, the possible targets of miR-140-3p were sought. Through identification, PPM1A was sponged by miR-140-3p. Philippe et al. attested that $\mathrm{miR}-19 \mathrm{a} / \mathrm{b}$ could act as negative regulators in RA-FLSs by controlling TLR2 expression [35]. Nevertheless, whether miR-140-3p could regulate PPM1A level to affect RA progression is still ill-defined. In different cancers, PPM1A has been evinced to serve as a tumor suppresser or promoter [36, 37]. However, the precise function of PPM1A in RA progression has not been expounded. Lee et al. has disclosed that PPM1A was highly expressed in RA, and PPM1A expression was positively correlated with pro-inflammatory cytokine TNF level in RA synovial fluid [17]. In this study, the data showed that PPM1A enrichment was elevated in synovial tissue from RA patients and RA-FLSs, manifesting that PPM1A might be involved in RA progression. Interestingly, the rescue experiments indicated that PPM1A overexpression could abolish the impacts of miR-140-3p introduction on cell progression and inflammation in RA-FLSs, hinting that miR-140-3p could regulate the malignant development of RA via targeting PPM1A, which was parallel with the previous report [35]. Moreover, circMAPK9 was attested to positively regulate PPM1A expression by the crosstalk of miR-1403p. Collectively, these data indicated that circMAPK 9 might promote RA progression by regulating miR-1403p/PPM1A axis.

This research conducted the in vitro experiments using the primary RA-FLSs, which represented the physiological function of RA patients. Furthermore, the involvement of circMAPK9/miR-140-3p/PPM1A network in RA-FLSs dysfunction was firstly confirmed, implying the significance and clinical expectation of this axis in RA advancement and therapy. Nevertheless, some limitations were still subsistent in the current study. For example, a larger number of RA patients and animal studies are needed in a further study in consideration of the limited number of patients and the restriction of in vitro experiments in this study. Besides, nanotechnology plays significant role in the area of bone-related therapy through providing attractive carrier options for delivery of therapeutic agents $[38,39]$. The progressive damage of articular bone and cartilage was developed in RA patients, which might cause disability over time [40]. Recent evidence has suggested that bioengineered composite scaffolds and magnetic nanoparticles are effective promising therapeutic tools for RA remedy [41, 42]. Therefore, the combination of nanotechnology and molecular targeted drugs might be a most effective method for RA treatment.

\section{Conclusion}

In conclusion, circMAPK9 interference constrained RAFLSs proliferation, migration, invasion, inflammatory response, and expedited apoptosis possibly by enhancing miR-140-3p and lessening PPM1A expression. Our study first elucidated the circMAPK9/miR-140-3p/PPM1A regulatory network in RA-FLSs, offering a new perception about RA-FLSs progression, and offering a novel possible target for RA therapy.

\section{Abbreviations}

RA: Rheumatoid arthritis; FLSs: Fibroblast-like synoviocytes; PPM1A: Protein phosphatase magnesium-dependent 1A; ELISA: Enzyme-linked

immunosorbent assay; circMAPK9: CircRNA mitogen-activated protein kinase; PBMCs: Peripheral blood mononuclear cells; FBS: Fetal bovine serum; qRTPCR: Quantitative real-time polymerase chain reaction; siRNA: Small interfering RNA; DMSO: Dimethyl sulfoxide; FITC: Fluorescein isothiocyanate; WT: Wild-type; SD: Standard deviation 


\section{Acknowledgement}

None.

\section{Authors' contributions}

ZL was responsible for drafting the manuscript. $\mathrm{ZL}, \mathrm{SC}$, and $\mathrm{XC}$ contributed to the analysis and interpretation of data. SC and XC contributed in the data collection. The authors read and approved the final manuscript.

\section{Funding}

None.

\section{Availability of data and materials}

The data that support the findings of this study are available from the corresponding author upon reasonable request.

\section{Declarations}

\section{Ethics approval and consent to participate}

The hospital's Institutional Review Board approved the current study.

\section{Consent for publication}

Not applicable.

\section{Competing interests}

The authors declare that they have no competing interest.

Received: 12 January 2021 Accepted: 10 June 2021

Published online: 21 June 2021

\section{References}

1. Smolen JS, Aletaha D, Barton A, Burmester GR, Emery P, Firestein GS, et al. Rheumatoid arthritis. Nat Rev Dis Primers. 2018;4(1):18001. https://doi.org/1 0.1038/nrdp.2018.1.

2. Scott DL, Wolfe F, Huizinga TW. Rheumatoid arthritis. Lancet. 2010; 376(9746):1094-108. https://doi.org/10.1016/S0140-6736(10)60826-4.

3. Harre U, Schett G. Cellular and molecular pathways of structural damage in rheumatoid arthritis. Semin Immunopathol. 2017;39(4):355-63. https://doi. org/10.1007/s00281-017-0634-0

4. Karami J, Aslani S, Tahmasebi MN, Mousavi MJ, Sharafat Vaziri A, Jamshidi A, et al. Epigenetics in rheumatoid arthritis; fibroblast-like synoviocytes as an emerging paradigm in the pathogenesis of the disease. Immunol Cell Biol. 2020;98(3):171-86. https://doi.org/10.1111/imcb.12311.

5. Bullock J, Rizvi SAA, Saleh AM, Ahmed SS, Do DP, Ansari RA, et al. Rheumatoid arthritis: a brief overview of the treatment. Med Princ Pract. 2018;27(6):501-7. https://doi.org/10.1159/000493390.

6. Kristensen LS, Andersen MS, Stagsted LW, Ebbesen KK, Hansen TB, Kjems J. The biogenesis, biology and characterization of circular RNAs. Nat Rev Genet. 2019;20(11):675-91. https://doi.org/10.1038/s41576-019-0158-7.

7. Memczak S, Jens M, Elefsinioti A, Torti F, Krueger J, Rybak A, et al. Circular RNAs are a large class of animal RNAs with regulatory potency. Nature. 2013;495(7441):333-8. https://doi.org/10.1038/nature11928.

8. Zhang Z, Yang T, Xiao J. Circular RNAs: promising biomarkers for human diseases. EBioMedicine. 2018;34:267-74. https://doi.org/10.1016/j.ebiom.201 8.07.036.

9. Xia $X$, Tang $X$, Wang S. Roles of CircRNAs in autoimmune diseases. Front Immunol. 2019;10:639. https://doi.org/10.3389/fimmu.2019.00639.

10. Wen J, et al. RNA-seq reveals the circular RNA and miRNA expression profile of peripheral blood mononuclear cells in patients with rheumatoid arthritis. Biosci Rep. 2020;40(4):BSR20193160. https://doi.org/10.1042/BSR20193160.

11. Ardekani AM, Naeini MM. The role of microRNAs in human diseases. Avicenna J Med Biotechnol. 2010;2(4):161-79.

12. Hansen TB, Jensen TI, Clausen BH, Bramsen JB, Finsen B, Damgaard CK, et al. Natural RNA circles function as efficient microRNA sponges. Nature. 2013; 495(7441):384-8. https://doi.org/10.1038/nature11993.

13. Evangelatos G, Fragoulis GE, Koulouri V, Lambrou GI. MicroRNAs in rheumatoid arthritis: from pathogenesis to clinical impact. Autoimmun Rev. 2019:18(11):102391. https://doi.org/10.1016/j.autrev.2019.102391.

14. Tavasolian F, Abdollahi E, Rezaei R, Momtazi-borojeni AA, Henrotin Y, Sahebkar A. Altered expression of MicroRNAs in rheumatoid arthritis. J Cell Biochem. 2018;119(1):478-87. https://doi.org/10.1002/jcb.26205.
15. Churov AV, Oleinik EK, Knip M. MicroRNAs in rheumatoid arthritis: altered expression and diagnostic potential. Autoimmun Rev. 2015;14(11):1029-37. https://doi.org/10.1016/j.autrev.2015.07.005.

16. Peng JS, Chen SY, Wu CL, Chong HE, Ding YC, Shiau AL, et al. Amelioration of experimental autoimmune arthritis through targeting of synovial fibroblasts by intraarticular delivery of microRNAs 140-3p and 140-5p. Arthritis Rheumatol. 2016;68(2):370-81. https://doi.org/10.1002/art.39446.

17. Lee B, Song YS, Rhodes C, Goh TS, Roh JS, Jeong H, et al. Protein phosphatase magnesium-dependent $1 \mathrm{~A}$ induces inflammation in rheumatoid arthritis. Biochem Biophys Res Commun. 2020;522(3):731-5. https://doi.org/10.1016/j.bbrc.2019.11.112

18. Zhang $Y, X u Y Z$, Sun N, Liu JH, Chen FF, Guan XL, et al. Long noncoding RNA expression profile in fibroblast-like synoviocytes from patients with rheumatoid arthritis. Arthritis Res Ther. 2016;18(1):227. https://doi.org/10.11 86/s13075-016-1129-4.

19. Bartok B, Firestein GS. Fibroblast-like synoviocytes: key effector cells in rheumatoid arthritis. Immunol Rev. 2010;233(1):233-55. https://doi.org/1 0.1111/j.0105-2896.2009.00859.x.

20. Nygaard G, Firestein GS. Restoring synovial homeostasis in rheumatoid arthritis by targeting fibroblast-like synoviocytes. Nat Rev Rheumatol. 2020; 16(6):316-33. https://doi.org/10.1038/s41584-020-0413-5.

21. Wang J, et al. Non-coding RNAs in rheumatoid arthritis: from bench to bedside. Front Immunol. 2019;10:3129.

22. Zhou Z, Sun B, Huang S, Zhao L. Roles of circular RNAs in immune regulation and autoimmune diseases. Cell Death Dis. 2019;10(7):503. https:// doi.org/10.1038/s41419-019-1744-5.

23. Zhong S, Ouyang Q, Zhu D, Huang Q, Zhao J, Fan M, et al. Hsa_circ 0088036 promotes the proliferation and migration of fibroblast-like synoviocytes by sponging miR-140-3p and upregulating SIRT 1 expression in rheumatoid arthritis. Mol Immunol. 2020;125:131-9. https://doi.org/10.101 6/j.molimm.2020.07.004.

24. Noack M, Miossec P. Selected cytokine pathways in rheumatoid arthritis. Semin Immunopathol. 2017;39(4):365-83. https://doi.org/10.1007/s00281-01 7-0619-z.

25. Mateen S, Zafar A, Moin S, Khan AQ, Zubair S. Understanding the role of cytokines in the pathogenesis of rheumatoid arthritis. Clin Chim Acta. 2016; 455:161-71. https://doi.org/10.1016/j.cca.2016.02.010.

26. Kulcheski FR, Christoff AP, Margis R. Circular RNAs are miRNA sponges and can be used as a new class of biomarker. J Biotechnol. 2016;238:42-51. https://doi.org/10.1016/j.jbiotec.2016.09.011.

27. Hong $W$, et al. The effects of microRNAs on key signalling pathways and epigenetic modification in fibroblast-like synoviocytes of rheumatoid arthritis. Mediators Inflamm. 2018;2018:9013124.

28. Philippe L, Alsaleh G, Pichot A, Ostermann E, Zuber G, Frisch B, et al. MiR20a regulates ASK1 expression and TLR4-dependent cytokine release in rheumatoid fibroblast-like synoviocytes. Ann Rheum Dis. 2013;72(6):1071-9. https://doi.org/10.1136/annrheumdis-2012-201654.

29. Wang $Y$, et al. miR-140-3p inhibits bladder cancer cell proliferation and invasion by targeting FOXQ1. Aging (Albany NY). 2020;12(20):20366-79.

30. Chen MS, Lin CH, Huang LY, Qiu XM. CircRNA SMARCC1 sponges MiR-140$3 p$ to regulate cell progression in colorectal cancer. Cancer Manag Res. 2020;12:4899-910. https://doi.org/10.2147/CMAR.S254185

31. Yin CM, Suen WCW, Lin S, Wu XM, Li G, Pan XH. Dysregulation of both miR140-3p and miR-140-5p in synovial fluid correlate with osteoarthritis severity. Bone Joint Res. 2017:6(11):612-8. https://doi.org/10.1302/2046-3758. 611.BJR-2017-0090.R1.

32. Chen Y, Li Y, Zheng G, Zhou P. Construction and analysis of macrophage infiltration related circRNA-miRNA-mRNA regulatory networks in hepatocellular carcinoma. PeerJ. 2020;8:e10198. https://doi.org/10.7717/ peerj.10198.

33. Xu Q, Jia X, Wu Q, Shi L, Ma Z, Ba N, et al. Esomeprazole affects the proliferation, metastasis, apoptosis and chemosensitivity of gastric cancer cells by regulating InCRNA/circRNA-miRNA-mRNA ceRNA networks. Oncol Lett. 2020;20(6):329. https://doi.org/10.3892/ol.2020.12193.

34. Zhang J, Liu Y, Shi G. The circRNA-miRNA-mRNA regulatory network in systemic lupus erythematosus. Clin Rheumatol. 2021:40(1):331-9. https://doi. org/10.1007/s10067-020-05212-2.

35. Philippe L, Alsaleh G, Suffert G, Meyer A, Georgel P, Sibilia J, et al. TLR2 expression is regulated by microRNA miR-19 in rheumatoid fibroblast-like synoviocytes. J Immunol. 2012:188(1):454-61. https://doi.org/10.4049/ jimmunol.1102348. 
36. Hong Y, Liangliang G, Biying Y, Yishan D. PPM1A suppresses the proliferation and invasiveness of RCC cells via Smad2/3 signaling inhibition. J Recept Signal Transduct Res. 2021;41(3):245-54. https://doi.org/10.1080/1 0799893.2020.1806316.

37. Wu SK, Wang BJ, Yang Y, Feng XH, Zhao XP, Yang DL. Expression of PTEN, PPM1A and P-Smad2 in hepatocellular carcinomas and adjacent liver tissues. World J Gastroenterol. 2007;13(34):4554-9. https://doi.org/10.3748/ wjg.v13.i34.4554

38. Maghsoudlou MA, Nassireslami E, Saber-Samandari S, Khandan A. Bone regeneration using bio-nanocomposite tissue reinforced with bioactive nanoparticles for femoral defect applications in medicine. Avicenna J Med Biotechnol. 2020;12(2):68-76.

39. Esmaeili S, Akbari Aghdam H, Motififard M, Saber-Samandari S, Montazeran AH, Bigonah M, et al. A porous polymeric-hydroxyapatite scaffold used for femur fractures treatment: fabrication, analysis, and simulation. Eur J Orthop Surg Traumatol. 2020;30(1):123-31. https://doi.org/10.1007/s00590-019-0253 $0-3$

40. Shim JH, Stavre Z, Gravallese EM. Bone loss in rheumatoid arthritis: basic mechanisms and clinical implications. Calcif Tissue Int. 2018;102(5):533-46. https://doi.org/10.1007/s00223-017-0373-1.

41. Zhao Y, Gao C, Liu H, Liu H, Feng Y, Li Z, et al. Infliximab-based self-healing hydrogel composite scaffold enhances stem cell survival, engraftment, and function in rheumatoid arthritis treatment. Acta Biomater. 2021;121:653-64. https://doi.org/10.1016/j.actbio.2020.12.005.

42. Liu Y, Cao F, Sun B, Bellanti JA, Zheng SG. Magnetic nanoparticles: a new diagnostic and treatment platform for rheumatoid arthritis. J Leukoc Biol. 2021;109(2):415-24. https://doi.org/10.1002/JLB.5MR0420-008RR.

\section{Publisher's Note}

Springer Nature remains neutral with regard to jurisdictional claims in published maps and institutional affiliations.

Ready to submit your research? Choose BMC and benefit from:

- fast, convenient online submission

- thorough peer review by experienced researchers in your field

- rapid publication on acceptance

- support for research data, including large and complex data types

- gold Open Access which fosters wider collaboration and increased citations

- maximum visibility for your research: over $100 \mathrm{M}$ website views per year

At BMC, research is always in progress.

Learn more biomedcentral.com/submissions 